\section{Atividade física em profissionais de saúde do Sul e Nordeste do Brasil}

\author{
Physical activity among health professionals from \\ South and Northeast Brazil
}

\footnotetext{
1 Programa de Pós-graduação em Educação Física, Universidade Federal de Santa Catarina, Florianópolis, Brasil. 2 Departamento de Medicina Social, Universidade Federal de Pelotas, Pelotas, Brasil.

3 Centro de Ciências da Vida e da Saúde, Universidade Católica de Pelotas, Pelotas, Brasil

4 Faculdade de Enfermagem e Obstetrícia, Universidade Federal de Pelotas, Pelotas, Brasil.

5 Programa de Pós-graduação em Epidemiologia, Universidade Federal de Pelotas, Pelotas, Brasil.

Correspondência F. C. V. Siqueira Programa de Pós-graduação em Educação Física, Universidade Federal de Santa Catarina.

C. P. 476, Campus Universitário Trindade, Florianópolis, SC 88040-900, Brasil. fcvsiqueira@uol.com.br
}

\begin{abstract}
This cross-sectional study analyzed 240 primary healthcare units, linked either to the traditional primary care model or the Family Health Program (FHP), involving a total of 3,347 health professionals who answered the International Physical Activity Questionnaire. Prevalence of sedentary lifestyle $(<150$ minutes of physical activity per week) was 27.5\% (95\%CI: 25.9-29.0). Sedentary lifestyle was more frequent among health professionals in the traditional model, with the difference explained by the presence of community health agents, whose work only involves extensive physical activity in the FHP model. Sedentary health professionals were proportionally more common in large cities with better socioeconomic conditions. The study highlights the importance of education for health professionals aimed at orientation and practice in physical activities and collaboration between primary healthcare workers and physical education teachers.

Motor Activity; Health Personnel; Family Health Program
\end{abstract}

Fernando Carlos Vinholes Siqueira ${ }^{1}$

Markus Vinícius Nahas ${ }^{1}$

Luiz Augusto Facchini 2

Roberto Xavier Piccini 2

Elaine Tomasi 3

Elaine Thumé 4

Denise Silva da Silveira 2

Pedro C. Hallal 5

\section{Introdução}

O sedentarismo é um dos fatores de risco para agravos à saúde mais prevalentes na população mundial e brasileira, sendo identificado atualmente como um importante problema de saúde pública nos mais diferentes segmentos 1,2. Estudos têm mostrado que a atividade física pode cumprir um importante papel preventivo e terapêutico e deve, portanto, ser parte integrante das práticas terapêuticas em saúde ${ }^{3,4}$. Essa relevância quanto ao papel preventivo da atividade física em relação a diferentes populações e aos profissionais de saúde que trabalham na atenção básica cresce com o aumento da expectativa de vida, da proporção de idosos na população e da prevalência de doenças crônicas 5 . Estimase que, com as mudanças decorrentes da transição demográfica, com os avanços da medicina e com a maior expectativa de vida, a população de adultos e idosos continue aumentando, sendo esperado um acréscimo de $300 \%$ na proporção de idosos no mundo até o ano de 20256 .

Nesta perspectiva, ganha em importância a atividade dos profissionais de saúde da rede básica de saúde para educar e orientar à prática de atividade física, modificando e melhorando seu comportamento em relação a um estilo de vida saudável e suas orientações para a promoção da saúde, à prevenção dos riscos relacionados a um estilo de vida sedentário, ao tratamento e reabilitação de agravos na população ? 
Um estudo realizado por Ribeira et al. 8, em 2005, mostrou que a promoção de atividade física pelos profissionais de saúde é oportunista, focada em pacientes selecionados, usando geralmente recomendações e altamente dependente do interesse pessoal. Outro estudo verificou se os médicos alcançavam as recomendações em termos de atividade física e concluiu que, embora as caminhadas no trabalho supram uma proporção substancial das necessidades diárias para os médicos, ainda é necessário o engajamento em horas adicionais de atividade física para que alcancem as recomendações 9. Assim, pode-se argumentar que profissionais de saúde deveriam manter hábitos de vida saudáveis, pois dessa forma, seria mais fácil exercer a função de estimular a prática de atividade física no dia-a-dia profissional 10,11 . No entanto, ainda é escasso o conhecimento sobre o comportamento em termos de atividade física em uma população específica de profissionais de saúde, particularmente na atenção básica do Brasil 12 .

Este estudo tem como objetivo descrever a prevalência de sedentarismo em profissionais de saúde da atenção básica nas regiões Sul e Nordeste do Brasil, e verificar fatores associados ao sedentarismo nestes profissionais.

\section{Métodos}

Um estudo de delineamento transversal identificou uma amostra de 4.749 profissionais de saúde que responderam ao instrumento de pesquisa. Eram trabalhadores das unidades básicas de saúde (UBS) de 41 municípios com mais de 100 mil habitantes dos Estados do Rio Grande do Sul e Santa Catarina na Região Sul, Alagoas, Pernambuco, Paraíba, Rio Grande do Norte e Piauí na Região Nordeste do Brasil 13. Os municípios incluídos no estudo compõem os Lotes 2 (Sul e Nordeste) do Estudo de Linha de Base do Projeto de Expansão e Consolidação do Saúde da Família (PROESF) 7.

Uma amostra aleatória de 240 UBS foi sorteada com diferentes modalidades de atenção básica - Programa Saúde da Família (PSF) e Tradicional. As UBS do PSF foram selecionadas de acordo com o tempo de implantação, PSF Pré-PROESF e Pós-PROESF. As UBS tradicionais foram consideradas aquelas que se caracterizavam pela presença na equipe de médicos especialistas (clínico, pediatra e gineco-obstetra), enfermeiro, auxiliar de enfermagem e pessoal administrativo. Muitas dessas unidades contam com o apoio esporádico de outras especialidades profissionais (dentista, nutricionista, fisioterapeuta) e mesmo médicas. A população atendida caracteriza-se por ser es- pontânea ou em razão do encaminhamento de outros serviços. As unidades de PSF, ao contrário, são formadas por equipes de saúde da família, que incluem médico generalista, enfermeiro, auxiliares de enfermagem e agentes comunitários, responsáveis pelo cadastramento e acompanhamento da população residente na área de abrangência do serviço 14 .

O sorteio das UBS foi proporcional ao tamanho da rede básica de cada município. A seleção da amostra nos municípios foi proporcional à capacidade instalada de sua rede básica. Na região Sul, obteve-se uma amostra de 69 UBS de PSF e 51 tradicionais. No Nordeste, a amostra foi constituída de 79 UBS de PSF e 41 tradicionais. Todos os profissionais das 240 UBS foram convidados a participar do estudo, incluindo médicos, enfermeiros, nutricionistas, odontólogos, psicólogos, técnicos e auxiliares de enfermagem, recepcionistas e agentes comunitários de saúde. A amostra final de 3.347 profissionais de saúde permitiu que fossem estimados valores de prevalência de $10 \%$ ou maiores, com margem de erro de dois pontos percentuais, nível de $95 \%$ de confiança e poder de $80 \%$. Com o tamanho de amostra estudado, houve possibilidade de detectar como estatisticamente significativas razões de prevalência próximas de 1,20 ou superiores na análise da amostra total. Quando a análise foi estratificada por região, foi possível detectar como estatisticamente significativas diferenças próximas de 1,40 ou superiores.

Os profissionais de saúde foram convidados a responder um questionário auto-aplicado, constituído de informações demográficas, sócio-econômicas, características do trabalho e situação de saúde. O nível de atividade física dos profissionais de saúde foi avaliado utilizando-se a versão curta do Questionário Internacional de Atividade Física (IPAQ) 15. Este instrumento avalia atividades físicas realizadas no tempo de lazer, deslocamentos, serviços domésticos e atividades ocupacionais. Um escore de atividade física em minutos por semana foi construído, somando-se os minutos despendidos em caminhada e atividades de intensidade moderada com os minutos despendidos em atividades de intensidade vigorosa multiplicados por dois. Tal estratégia visa considerar as diferentes intensidades de cada atividade e está de acordo com as recomendações atuais quanto à prática de atividade física 16,17 . Um escore abaixo de 150 minutos por semana foi o ponto de corte para classificar os profissionais como sedentários 15,16,17.

As análises descritivas incluíram cálculos de proporções e respectivos intervalos de 95\% de confiança (IC95\%). Na análise bruta, a prevalência de sedentarismo foi calculada para ca- 
da grupo das variáveis independentes, e o nível de significância foi testado usando os testes de Wald para heterogeneidade e tendência linear. As variáveis independentes incluídas nesta análise foram: (a) sexo; (b) idade (16 a 29 anos, 30 a 49 anos e 50 anos ou mais); (c) escolaridade (fundamental incompleto, fundamental completo, médio incompleto, médio completo, superior incompleto, superior completo e pós-graduação); (d) tabagismo (não e sim); (e) horas de trabalho cumpridas na UBS (até 20 horas, de 21 horas a 40 horas, e 40 horas ou mais); (f) tempo de trabalho na UBS (até 24 meses, 25 meses a 108 meses, 109 meses ou mais; a categorização escolhida coincide com a atenção tradicional, o inicio do PSF e com a data do investimento do PROESF); (h) regime de trabalho (trabalho precário ou não); (g) utilização de protocolos pelo profissional (sim ou não); (h) atividade profissional na UBS (médico, enfermeiro, outro profissional de nível superior, agente comunitário de saúde e outros profissionais de nível médio); (i) primeiro emprego (sim ou não); (j) outro emprego além da UBS (sim ou não); (k) auto-avaliação da qualidade de vida (muito ruim, ruim, nem ruim nem boa, boa, muito boa).

Trabalho foi considerado precário se realizado com a ausência de direitos trabalhistas e previdenciários consagrados na lei e a ausência de concurso ou processo seletivo público para cargo permanente ou emprego público no SUS 18 . A utilização de protocolos por sua vez, foi considerada como uso de procedimentos que sumarizam as evidências disponíveis na literatura para a definição de rotinas diagnósticas, de tratamento e de regulação da demanda de exames e procedimentos 19 .

A análise ajustada foi realizada por regressão de Poisson com estimativas robustas de variância 20 , com cálculo de razões de prevalência ajustadas, IC95\%, e valores de significância usando os mesmos testes descritos anteriormente. Todas as análises ajustadas levaram em consideração o desenho amostral, e foi utilizado um modelo hierárquico de análise 7,21. O modelo incluiu as variáveis independentes "a-d" no primeiro nível. As demais variáveis foram consideradas no nível intermediário em relação ao desfecho sedentarismo. Cada uma das variáveis foi ajustada para possíveis fatores de confusão em relação a aquelas do mesmo nível e de níveis anteriores. Todas variáveis com $\mathrm{p}<0,20$ foram mantidas no modelo para eventual controle de fatores de confusão.

O Comitê de Ética da Faculdade de Medicina da Universidade Federal de Pelotas aprovou o protocolo do estudo, e obteve-se consentimento informado de todos os participantes. Detalhes sobre o projeto e a metodologia utilizada podem ser revisados em outras publicações do grupo 7,13 .

\section{Resultados}

Um total de 4.749 profissionais de saúde foi localizado nas UBS amostradas. Destes, 3.347 responderam às questões relacionadas à atividade física, o que resultou em uma taxa de resposta de $70,5 \%$. Entre os respondentes, $79,3 \%$ foram mulheres, enquanto entre os não respondentes a proporção foi de $85,4 \%$. A média de idade foi de 37,0 anos entre os que responderam ao instrumento e de 37,4 anos entre os que não responderam. Em termos de atividade profissional, 33\% dos respondentes e 33,3\% dos não respondentes eram agentes comunitários de saúde. A Tabela 1 descreve a amostra de profissionais conforme as variáveis independentes incluídas no estudo. A proporção de mulheres foi maior na totalidade da amostra e em ambas as regiões. A faixa etária entre 30 e 49 anos foi a mais prevalente tanto no Sul como no Nordeste, e a média da idade foi de 37,2 (desvio-padrão-DP: 10,4).

A prevalência de sedentarismo em profissionais da saúde das duas regiões pesquisadas foi de 27,5\% (IC95\%: 25,9-29,0). Entre os profissionais da região Sul, o percentual foi de $28,2 \%$ (IC95\%: 25,7-30,7), enquanto para os profissionais da região Nordeste, o valor foi $27 \%$ (IC95\%: 25,1-28,9).

O sedentarismo foi significativamente maior ( $\mathrm{p}=0,01)$ naqueles que trabalhavam no modelo tradicional (30,6\%) em relação àqueles vinculados ao PSF. Ao excluirmos os agentes comunitários de saúde da análise, que estão presentes no processo de trabalho do modelo PSF, as prevalências de sedentarismo foram similares de acordo com o modelo de atenção à saúde (Tabela 2). Os resultados também foram similares nas regiões Sul e Nordeste.

Os profissionais de saúde dos municípios de fora da Região Metropolitana de Recife foram os mais sedentários (28,7\%), com uma chance $23 \%$ maior para o sedentarismo em relação aos da região metropolitana $(p=0,01)$. No Sul, esta diferença não foi verificada; aqueles que trabalham na Região Metropolitana de Porto Alegre tiveram um percentual de sedentarismo (31\%) similar ao encontrado nos que trabalham fora da região metropolitana (27\%). Para os estados pesquisados, as prevalências de sedentarismo entre os profissionais foram de 33,7\% na Paraíba, 33,4\% no Rio Grande do Norte, 28,9\% no Rio Grande do Sul, 25,8\% em Santa Catarina, 25,3\% no Piauí, 24,7\% em Alagoas e 23,6\% em Pernambuco. 
Descrição das variáveis independentes na amostra de profissionais de saúde de unidades básicas de saúde (UBS) de municípios das regiões Sul e Nordeste do Brasil, 2005.

\begin{tabular}{|c|c|c|c|c|c|c|}
\hline \multirow[t]{2}{*}{ Variáveis } & \multicolumn{2}{|c|}{ Região Sul } & \multicolumn{2}{|c|}{ Região Nordeste } & \multicolumn{2}{|c|}{ Total } \\
\hline & $\%$ & n & $\%$ & n & $\%$ & $\mathbf{N}$ \\
\hline Sexo & & 1.228 & & 2.089 & & 3.317 \\
\hline Masculino & 20,0 & & 21,1 & & 20,7 & \\
\hline Feminino & 80,0 & & 78,9 & & 79,3 & \\
\hline Idade (anos) & & 1.227 & & 2.087 & & 3.314 \\
\hline $16-29$ & 29,2 & & 26,1 & & 27,2 & \\
\hline $30-49$ & 58,3 & & 59,6 & & 59,1 & \\
\hline 50 ou + & 12,5 & & 14,3 & & 13,7 & \\
\hline Escolaridade & & 1.226 & & 2.097 & & 3.323 \\
\hline Fundamental incompleto & 3,1 & & 5,8 & & 4,8 & \\
\hline Fundamental completo & 4,6 & & 4,9 & & 4,8 & \\
\hline Médio incompleto & 8,7 & & 6,4 & & 7,3 & \\
\hline Médio completo & 33,8 & & 45,3 & & 41,0 & \\
\hline Superior incompleto & 7,6 & & 7,3 & & 7,4 & \\
\hline Superior completo & 16,0 & & 14,9 & & 15,3 & \\
\hline Pós-graduação & 26,2 & & 15,4 & & 19,5 & \\
\hline Tabagismo & & 1.134 & & 1.992 & & 3.126 \\
\hline Não & 84,0 & & 91,0 & & 88,5 & \\
\hline Sim & 16,0 & & 9,0 & & 11,5 & \\
\hline Horas de trabalho cumpridas & & 1.187 & & 2.043 & & 3.230 \\
\hline Até 20 & 17,7 & & 11,6 & & 13,8 & \\
\hline $21-40$ & 80,4 & & 86,2 & & 84,0 & \\
\hline 41 ou mais & 1,9 & & 2,2 & & 2,2 & \\
\hline Tempo de trabalho na UBS (meses) & & 1.211 & & 2.061 & & 3.272 \\
\hline Até 24 & 63,5 & & 45,4 & & 52,1 & \\
\hline $25-108$ & 29,1 & & 41,4 & & 36,8 & \\
\hline 109 ou + & 7,4 & & 13,2 & & 11,1 & \\
\hline Regime de trabalho precarizado & & 1.199 & & 2.041 & & 3.240 \\
\hline Não & 70,1 & & 57,5 & & 62,1 & \\
\hline $\operatorname{Sim}$ & 29,9 & & 42,5 & & 37,9 & \\
\hline Utilização de protocolos pelo profissional & & 1.181 & & 2.031 & & 3.212 \\
\hline Não & 62,4 & & 50,0 & & 54,6 & \\
\hline $\operatorname{Sim}$ & 37,6 & & 50,0 & & 45,4 & \\
\hline Atividade profissional na UBS & & 1.222 & & 2.069 & & 3.291 \\
\hline Médico & 19,4 & & 9,5 & & 13,2 & \\
\hline Enfermeiro & 10,6 & & 7,2 & & 8,5 & \\
\hline Outros de nível superior & 9,3 & & 8,9 & & 9,0 & \\
\hline Agentes comunitários de saúde & 25,9 & & 37,2 & & 33,0 & \\
\hline Outros de nível médio & 34,8 & & 37,2 & & 36,3 & \\
\hline Primeiro emprego & & 1.216 & & 2.092 & & 3.308 \\
\hline Não & 82,9 & & 68,5 & & 73,8 & \\
\hline $\operatorname{Sim}$ & 17,1 & & 31,5 & & 26,4 & \\
\hline Outro emprego além da UBS & & 1.218 & & 2.085 & & 3.303 \\
\hline Não & 77,1 & & 78,3 & & 77,8 & \\
\hline $\operatorname{Sim}$ & 22,9 & & 21,7 & & 22,2 & \\
\hline Auto-avaliação da qualidade de vida & & 1.220 & & 2.096 & & 3.316 \\
\hline Muito ruim & 0,5 & & 0,3 & & 0,4 & \\
\hline Ruim & 2,2 & & 2,8 & & 2,6 & \\
\hline Nem ruim nem boa & 16,4 & & 24,3 & & 21,4 & \\
\hline Boa & 63,9 & & 62,7 & & 63,2 & \\
\hline Muito boa & 17,0 & & 9,9 & & 12,4 & \\
\hline
\end{tabular}


Prevalência de sedentarismo e intervalo de 95\% de confiança (IC95\%) das variáveis de contexto na amostra total de profissionais de saúde e por região Sul e Nordeste do Brasil, 2005.

\begin{tabular}{|c|c|c|c|c|c|c|c|c|c|}
\hline \multirow[t]{2}{*}{ Variáveis } & \multicolumn{3}{|c|}{ Região Sul } & \multicolumn{3}{|c|}{ Região Nordeste } & \multicolumn{3}{|c|}{ Total } \\
\hline & $\%$ & $\mathrm{IC} 95 \%$ & $p$ & $\%$ & IC95\% & $\mathrm{p}$ & $\%$ & IC95\% & $\mathrm{p}$ \\
\hline Modelo & & & 0,37 & & & 0,005 & & & 0,01 \\
\hline Tradicional & 30,5 & $26,1-34,9$ & & 30,7 & $27,4-34,0$ & & 30,6 & $27,9-33,2$ & \\
\hline PSF Pré-PROESF & 25,9 & $21,2-30,6$ & & 25,8 & $23,0-28,6$ & & 25,8 & $23,4-28,2$ & \\
\hline PSF Pós-PROESF & 27,9 & $23,8-31,9$ & & 23,5 & $19,5-27,4$ & & 25,7 & $22,9-28,6$ & \\
\hline Modelo sem agente comunitário de saúde & & & 0,06 & & & 0,09 & & & 0,68 \\
\hline Tradicional & 33,1 & $30,1-38,2$ & & 31,2 & $26,5-35,3$ & & 34,0 & $30,9-37,0$ & \\
\hline PSF Pré-PROESF & 31,8 & $29,9-37,0$ & & 26,7 & $22,4-30,2$ & & 33,3 & $30,0-36,6$ & \\
\hline PSF Pós-PROESF & 28,7 & $25,4-32,1$ & & 28,6 & $24,7-32,5$ & & 33,0 & $29,0-36,9$ & \\
\hline Região & & & 0,11 & & & 0,01 & & & 0,32 \\
\hline Metropolitana & 31,0 & $26,6-35,3$ & & 23,3 & $20,0-26,5$ & & 26,4 & $23,7-28,9$ & \\
\hline Não Metropolitana & 26,7 & $23,6-29,7$ & & 28,7 & $26,4-31,0$ & & 28,0 & $26,1-29,2$ & \\
\hline Estado & & & 0,31 & & & $<0,001$ & & & 0,002 \\
\hline RS & 28,9 & $26,0-31,8$ & & & - & & 28,9 & $26,0-31,8$ & \\
\hline SC & 25,8 & $20,7-30,1$ & & & - & & 25,8 & $20,7-30,9$ & \\
\hline$A L$ & & - & & 24,7 & $20,3-29,1$ & & 24,7 & $20,3-29,0$ & \\
\hline PB & & - & & 33,7 & $26,9-40,5$ & & 33,7 & $26,9-26,5$ & \\
\hline PE & & - & & 23,6 & $20,7-26,5$ & & 23,6 & $20,7-26,5$ & \\
\hline $\mathrm{PI}$ & & - & & 25,3 & $20,1-30,5$ & & 25,3 & $20,1-30,4$ & \\
\hline RN & & - & & 33,4 & $29,1-37,8$ & & 33,4 & $29,1-37,7$ & \\
\hline Porte do município (por mil habitantes) & & & 0,007 * & & & 0,006 * & & & 0,001 \\
\hline $100-200$ & 24,0 & $20,0-27,9$ & & 21,8 & $18,3-25,4$ & & 22,8 & $20,2-25,5$ & \\
\hline $200-500$ & 30,0 & $26,4-33,6$ & & 28,5 & $24,4-32,6$ & & 29,4 & $26,7-32,1$ & \\
\hline$>500$ & 33,8 & $25,7-41,9$ & & 28,8 & $26,2-31,5$ & & 29,4 & $26,9-31,9$ & * \\
\hline
\end{tabular}

PSF: Programa Saúde da Família; PROESF: Projeto de Expansão e Consolidação do Saúde da Família; RS: Rio Grande do Sul; SC: Santa Catarina; AL: Alagoas; PB: Paraíba; PE: Pernambuco; PI: Piauí; RN: Rio Grande do Norte.

* Teste de Wald para tendência.

O sedentarismo nos profissionais também apresentou diferenças significativas, tanto na amostra total quanto na estratificada por região, em função do porte populacional do município. Os profissionais foram sempre mais sedentários nos municípios com mais de 500 mil habitantes, tanto no Sul $(33,8 \%)$, quanto no Nordeste (28,8\%). Nos municípios com menos de $200 \mathrm{mil}$ habitantes as prevalências variaram de $24 \%$ no Sul a 21,8\% no Nordeste. Em municípios com população entre 200 e 500 mil habitantes, o sedentarismo oscilou entre $30 \%$ no Sul e $28,5 \%$ no Nordeste.

A Tabela 3 apresenta a associação bruta e ajustada entre sedentarismo e as variáveis independentes na amostra total. O sedentarismo esteve significativamente associado com o sexo masculino na análise bruta, porém perdeu significância estatística na análise ajustada. As variáveis, idade, tabagismo, tempo de trabalho na
UBS e primeiro emprego não estiveram associadas com sedentarismo nas análises.

Foi observada associação direta entre a prevalência de sedentarismo e escolaridade tanto na análise bruta como na ajustada. Trabalhar na UBS até 20 horas por semana mostrou maior chance para sedentarismo, tanto na análise bruta como na ajustada. Aqueles profissionais em regime de trabalho precário mostraram-se estatisticamente menos sedentários na análise bruta, no entanto, na análise ajustada, o valor perdeu significância.

Outra variável associada ao sedentarismo na análise bruta e ajustada foi o uso de protocolos: profissionais que usam os protocolos são menos sedentários que os demais. Ser médico esteve associado com sedentarismo somente na análise bruta, mas a atividade profissional de agentes comunitários de saúde esteve associada com sedentarismo tanto na análise bruta quanto na ajustada. Associações com sedentarismo 
Prevalência de sedentarismo conforme as variáveis independentes, análise bruta e ajustada da amostra de profissionais da saúde de unidades básicas de saúde (UBS) de municípios das regiões Sul e Nordeste do Brasil, 2005.

\begin{tabular}{|c|c|c|c|c|c|}
\hline \multirow[t]{2}{*}{ Variáveis (nível) } & \multicolumn{3}{|c|}{ Análise bruta } & \multicolumn{2}{|c|}{ Análise ajustada } \\
\hline & $\%$ & RP (IC95\%) & $p$ & RP (IC95\%) & $p$ \\
\hline \multicolumn{3}{|l|}{ Sexo (1) } & $<0,001$ & & 0,18 \\
\hline Masculino & 31,5 & 1,00 & & 1,00 & \\
\hline Feminino & 26,4 & $0,84(0,74-0,95)$ & & $0,91(0,79-1,04)$ & \\
\hline \multicolumn{3}{|l|}{ Idade (anos) (1) } & 0,09 * & & 0,468 \\
\hline $16-29$ & 26,3 & 1,00 & & 1,00 & \\
\hline $30-49$ & 27,0 & $1,03(0,90-1,17)$ & & $0,97(0,84-1,11)$ & \\
\hline $50 \mathrm{ou}+$ & 35,5 & $1,24(1,04-1,47)$ & & $1,17(0,97-1,41)$ & \\
\hline \multicolumn{3}{|l|}{ Escolaridade (1) } & $<0,001$ * & & 0,002 * \\
\hline Fundamental incompleto & 25,8 & 1,00 & & 1,00 & \\
\hline Fundamental completo & 21,4 & $0,83(0,56-1,23)$ & & $1,10(0,71-1,73)$ & \\
\hline Médio incompleto & 19,5 & $0,76(0,52-1,09)$ & & $0,82(0,54-1,27)$ & \\
\hline Médio completo & 21,2 & $0,82(0,62-1,09)$ & & $0,93(0,66-1,30)$ & \\
\hline Superior incompleto & 261 & $1,01(0,72-1,42)$ & & $1,14(0,77-1,67)$ & \\
\hline Superior completo & 36,4 & $1,41(1,06-1,88)$ & & $1,39(0,97-2,00)$ & \\
\hline Pós-graduação & 39,1 & $1,52(1,15-2,00)$ & & $1,42(0,98-2,06)$ & \\
\hline \multicolumn{3}{|l|}{ Tabagismo (1) } & 0,64 & & 0,06 \\
\hline Não & 27,7 & 1,00 & & 1,00 & \\
\hline Sim & 28,8 & $1,04(0,88-1,24)$ & & $1,19(0,99-1,42)$ & \\
\hline \multicolumn{3}{|l|}{ Horas de trabalho cumpridas (2) } & $<0,001$ * & & 0,04 * \\
\hline Até 20 & 37,8 & 1,00 & & 1,00 & \\
\hline $21-40$ & 25,8 & $0,68(0,60-0,78)$ & & $0,81(0,68-0,97)$ & \\
\hline 40 ou mais & 30,4 & $0,80(0,55-1,17)$ & & $0,98(0,62-1,55)$ & \\
\hline \multicolumn{3}{|l|}{ Tempo de trabalho na UBS (meses) (2) } & 0,75 * & & 0,38 * \\
\hline Até 24 & 27,3 & 1,00 & & 1,00 & \\
\hline $25-108$ & 28,5 & $1,04(0,93-1,17)$ & & $1,03(0,90-1,18)$ & \\
\hline 109 ou + & 25,2 & $0,92(0,76-1,12)$ & & $0,84(0,66-1,07)$ & \\
\hline \multicolumn{3}{|l|}{ Regime de trabalho precarizado (2) } & $<0,001$ & & 0,07 \\
\hline Não & 29,7 & 1,00 & & 1,00 & \\
\hline Sim & 23,5 & $0,79(0,69-0,82)$ & & $0,88(0,77-1,01)$ & \\
\hline \multicolumn{3}{|c|}{ Utilização de protocolos pelo profissional (2) } & 0,05 & & 0,05 \\
\hline Não & 29,2 & 1,00 & & 1,00 & \\
\hline Sim & 26,0 & $0,89(0,80-1,00)$ & & $0,88(0,77-1,00)$ & \\
\hline \multicolumn{3}{|l|}{ Atividade profissional na UBS (2) } & $<0,001$ & & 0,47 \\
\hline Médico & 43,5 & 1,00 & & 1,00 & \\
\hline Enfermeiro & 36,0 & $0,83(0.68-1,00)$ & & $0,90(0,72-1,14)$ & \\
\hline Outros de nível superior & 34,7 & $0,80(0,66-0,96)$ & & $0,86(0,68-1,08)$ & \\
\hline Agentes comunitários de saúde & 14,9 & $0,34(0,29-0,41)$ & & $0,43(0,33-0,65)$ & \\
\hline Outros de nível médio & 28,9 & $0,66(0,58-0,76)$ & & $0,83(0,62-1,10)$ & \\
\hline \multicolumn{3}{|l|}{ Primeiro emprego (2) } & 0,29 & & 0,11 \\
\hline Não & 27,8 & 1,00 & & 1,00 & \\
\hline Sim & 25,9 & $0,93(0,82-1,06)$ & & $1,13(0,97-1,31)$ & \\
\hline \multicolumn{3}{|l|}{ Outro emprego além da UBS (2) } & $<0,001$ & & $<0,001$ \\
\hline Não & 23,9 & 1,00 & & 1,00 & \\
\hline $\operatorname{Sim}$ & 39,6 & $1,66(1,48-1,85)$ & & $1,36(1,17-1,59)$ & \\
\hline \multicolumn{3}{|c|}{ Auto-avaliação da qualidade de vida (2) } & $<0,001$ * & & $<0,001$ * \\
\hline Muito ruim & 41,7 & 1,00 & & 1,00 & \\
\hline Ruim & 49,4 & $1,19(0,59-2,40)$ & & $1,20(0,53-2,75)$ & \\
\hline Nem ruim nem boa & 28,6 & $0,69(0,35-1,35)$ & & $0,85(0,38-1,92)$ & \\
\hline Boa & 27,3 & $0,65(0,33-1,28)$ & & $0,80(0,36-1,81)$ & \\
\hline Muito boa & 21,5 & $0,52(0,26-1,03)$ & & $0,58(0,25-1,33)$ & \\
\hline
\end{tabular}

RP (IC95\%): razão de prevalência (intervalo de 95\% de confiança).

* Teste de Wald para tendência. 
foram verificadas também para as variáveis "outro emprego além do realizado na UBS" e "autoavaliação da qualidade de vida”. Possuir outro emprego e classificar como ruim ou muito ruim a qualidade de vida, mostraram-se fatores associados para sedentarismo.

Nas Tabelas 4 e 5, são apresentadas separadamente as análises de acordo com região e modelo de atenção básica. Os profissionais do sexo feminino do Sul apresentaram menores chances para sedentarismo de $22 \%$ na análise bruta, todavia sem significância na análise ajustada. Para os profissionais do Nordeste, não foi verificada associação com sexo. A idade esteve diretamente associada com sedentarismo no Nordeste, e os profissionais mais velhos apresentaram maiores chances, elevando $46 \%$ em comparação aos mais jovens ( $p=0,001$ ). Já na Região Sul, os profissionais mais velhos apresentaram menores chances para o sedentarismo de $22 \%$ ( $p=0,04)$ em comparação aos mais jovens.

A escolaridade esteve associada ao sedentarismo na Região Nordeste, e os profissionais com pós-graduação foram os mais sedentários. Já no Sul, esta associação perdeu significância na análise ajustada. O tabagismo mostrou-se associado ao sedentarismo nos profissionais do Sul, entretanto a associação não foi verificada no Nordeste.

A variável "horas de trabalho cumpridas na UBS" esteve associada com o sedentarismo para os profissionais da Região Nordeste, mas tal associação não foi verificada na Região Sul. A utilização de protocolos pelos profissionais se associou com sedentarismo no Nordeste, enquanto no Sul a associação não foi encontrada. Comparados aos médicos, os agentes comunitários de saúde são menos sedentários tanto na análise bruta quanto na ajustada, em ambas as regiões.

Em relação ao modelo de atenção, a escolaridade foi uma variável associada ao sedentarismo nos profissionais do modelo tradicional enquanto no modelo PSF esta variável não se mostrou associada. Regime de trabalho precário, no modelo PSF, apresentou associação estatisticamente limítrofe $(\mathrm{p}=0,06)$ com sedentarismo. A mesma associação não se confirmou no modelo tradicional na análise ajustada. Profissionais que estão trabalhando no primeiro emprego apresentaram maiores chances de sedentarismo no modelo PSF, enquanto esta associação não se evidenciou para os profissionais do modelo tradicional. A auto-avaliação da qualidade de vida como "ruim e muito ruim" revelou-se associada ao sedentarismo para os profissionais apenas do modelo PSF. No modelo tradicional, a auto-avaliação muito boa esteve associada com sedentarismo, tanto na análise bruta quanto na ajustada.

\section{Discussão}

A prevalência de sedentarismo em profissionais de saúde das 240 UBS de municípios com mais de 100 mil habitantes dos sete estados brasileiros estudados, foi de $27,5 \%$, sendo $31,5 \%$ nos homens e $26,4 \%$ nas mulheres. Nosso resultado foi mais baixo que o encontrado em um estudo realizado no México, com mulheres profissionais da área da saúde em 2005, que utilizou o mesmo instrumento (IPAQ) e encontrou uma prevalência de sedentarismo de $43 \%$. Tais resultados devem ser observados com cautela, visto as diferenças metodológicas na aplicação do estudo que podem explicar estas diferenças 12 .

Em relação aos estados, verificamos que os profissionais de saúde da Paraíba foram os mais sedentários. Esse resultado é consistente com os dados de outros estudos que identificam o sedentarismo entre população da área de abrangência como maior no Nordeste 22, e o estudo do Instituto Nacional de Câncer (INCA), que detectou a maior prevalência de sedentarismo em João Pessoa, de um total de 15 capitais analisadas 23 .

Os municípios considerados de médio porte são aqueles em que os profissionais foram mais sedentários. Em relação às regiões estudadas, verificou-se que os profissionais do Sul foram sempre mais sedentários, independentemente do porte municipal. Uma das hipóteses para este achado é a diferença de clima entre as regiões, fato que poderia aumentar ainda mais as dificuldades em relação à prática de atividade física por barreiras ambientais 24 .

Estudos sobre nível de atividade física em profissionais das UBS são ainda pouco encontrados na literatura. Neste estudo verificamos que as variáveis sócio-econômicas têm grande importância na determinação deste desfecho. Verificamos que quanto maior o nível sócio-econômico dos profissionais de saúde, maior o sedentarismo entre eles. A mesma associação foi descrita em um estudo populacional por Hallal et al. 16. A hipótese é que as práticas de deslocamento ativo, serviços domésticos pesados e atividade física pesada no trabalho sejam mais freqüentes entre os pobres, gerando um maior nível de atividade física total em comparação aos ricos, os quais apresentam maior prática de atividade física no lazer 16. Outro aspecto é que o nível econômico está fortemente associado ao tipo de atividade profissional, assim, os agentes comunitários de saúde, que são os profissionais mais ativos, são os de menor remuneração no âmbito da atenção básica.

Ainda nesse contexto, podemos salientar a associação encontrada para aqueles trabalhado- 
Prevalência de sedentarismo, análise bruta e ajustada para profissionais de saúde de unidades básicas de saúde (UBS) conforme as regiões Sul e Nordeste do Brasil, 2005.

\begin{tabular}{|c|c|c|c|c|c|c|c|c|c|c|}
\hline \multirow[t]{3}{*}{ Variáveis (nível) } & \multicolumn{5}{|c|}{ Região Sul } & \multicolumn{5}{|c|}{ Região Nordeste } \\
\hline & \multicolumn{3}{|c|}{ Análise bruta } & \multicolumn{2}{|c|}{ Análise ajustada } & \multirow[b]{2}{*}{$\%$} & \multicolumn{2}{|c|}{ Análise bruta } & \multicolumn{2}{|c|}{ Análise ajustada } \\
\hline & $\%$ & RP (IC95\%) & $\mathrm{p}$ & RP (IC95\%) & $p$ & & RP (IC95\%) & $p$ & RP (IC95\%) & $p$ \\
\hline Sexo (1) & & & 0,02 & & 0,31 & & & 0,11 & & 0,20 \\
\hline Masculino & 34,2 & 1,00 & & 1,00 & & 30,0 & 1,00 & & 1,00 & \\
\hline Feminino & 26,7 & $0,78(0,64-0,96)$ & & $0,89(0,71-1,12)$ & & 26,3 & $0,88(0,74-1,03)$ & & $0,90(0,76-1,06)$ & \\
\hline Idade (anos) (1) & & & 0,20 * & & 0,04 * & & & 0,002 * & & 0,01 * \\
\hline $16-29$ & 31,0 & 1,00 & & 1,00 & & 23,1 & 1,00 & & 1,00 & \\
\hline $30-49$ & 27,0 & $0,88(0,72-1,06)$ & & $0,80(0,65-1,00)$ & & 26,9 & $1,16(0,97-1,39)$ & & $1,12(0,93-1,35)$ & \\
\hline $50 \mathrm{ou}+$ & 27,3 & $0,88(0,65-1,19)$ & & $0,78(0,56-1,09)$ & & 35,1 & $1,52(1,22-1,89)$ & & $1,46(1,15-1,84)$ & \\
\hline Escolaridade (1) & & & $<0,001$ * & & $0,15 *$ & & & $<0,001$ & & 0,005 * \\
\hline Fundamental incompleto & 29,0 & 1,00 & & 1,00 & & 24,8 & 1,00 & & 1,00 & \\
\hline Fundamental completo & 17,9 & $0,62(0,29-1,31)$ & & $0,80(0,34-1,88)$ & & 23,3 & $0,94(0,59-1,50)$ & & $1,07(0,65-1,78)$ & \\
\hline Médio incompleto & 20,8 & $0,72(0,38-1,34)$ & & $0,73(0,35-1,50)$ & & 18,5 & $0,75(0,47-1,20)$ & & $0,82(0,49-1,38)$ & \\
\hline Médio completo & 23,0 & $0,79(0,47-1,35)$ & & $0,77(0,41-1,45)$ & & 20,4 & $0,82(0,59-1,15)$ & & $0,92(0,64-1,34)$ & \\
\hline Superior incompleto & 23,7 & $0,82(0,44-1,52)$ & & $0,85(0,42-1,73)$ & & 27,6 & $1,11(0,74-1,67)$ & & $1,23(0,79-1,92)$ & \\
\hline Superior completo & 35,2 & $1,22(0,71-2,07)$ & & $1,05(0,54-2,06)$ & & 37,1 & $1,50(1,06-2,11)$ & & $1,43(0,96-2,14)$ & \\
\hline Pós-graduação & 36,5 & $1,26(0,75-2,12)$ & & $1,15(0,58-2,28)$ & & 41,7 & $1,68(1,20-2,35)$ & & $1,44(0,95-2,16)$ & \\
\hline Tabagismo (1) & & & 0,16 & & 0,003 & & & 0,45 & & 0,74 \\
\hline Não & 27,4 & 1,00 & & 1,00 & & 27,8 & 1,00 & & 1,00 & \\
\hline Sim & 32,4 & $1,18(0,94-1,49)$ & & $1,44(1,13-1,84)$ & & 25,1 & $0,90(0,69-1,18)$ & & $0,95(0,71-1,27)$ & \\
\hline Horas de trabalho & & & $0,28^{\star}$ & & 0,73 * & & & $<0,001$ & & $<0,001$ \\
\hline \multicolumn{11}{|l|}{ cumpridas (2) } \\
\hline Até 20 & 31,4 & 1,00 & & 1,00 & & 43,5 & 1,00 & & 1,00 & \\
\hline $21-40$ & 27,5 & $0,87(0,70-1,09)$ & & $1,03(0,77-1,38)$ & & 24,9 & $0,57(0,49-0,68)$ & & $0,64(0,50-0,80)$ & \\
\hline 40 ou mais & 30,4 & $0,97(0,51-1,85)$ & & $1,30(0,68-2,49)$ & & 30,4 & $0,70(0,44-1,11)$ & & $0,86(0,51-1,45)$ & * \\
\hline $\begin{array}{l}\text { Tempo de trabalho na UBS } \\
\text { (meses) (2) }\end{array}$ & & & 0,58 * & & 0,53 * & & & 0,918 & & 0,71 * \\
\hline Até 24 & 28,1 & 1,00 & & 1,00 & & 26,6 & 1,00 & & 1,00 & \\
\hline $25-108$ & 29,6 & $1,05(0,86-1,28)$ & & $1,11(0,88-1,41)$ & & 28,0 & $1,05(0,90-1,22)$ & & $1,03(0,87-1,22)$ & \\
\hline 109 ou + & 22,2 & $0,79(0,53-1,18)$ & & $0,64(0,36-1,12)$ & & 26,0 & $0,98(0,78-1,23)$ & & $0,91(0,69-1,20)$ & \\
\hline \multicolumn{11}{|l|}{ precarizado (2) } \\
\hline Não & 28,8 & 1,00 & & 1,00 & & 30,4 & 1,00 & & 1,00 & \\
\hline Sim & 26,7 & $0,93(0,76-1,14)$ & & $0,91(0,73-1,15)$ & & 22,1 & $0,73(0,63-0,85)$ & & $0,89(0,75-1,05)$ & \\
\hline $\begin{array}{l}\text { Utilização de protocolos } \\
\text { pelo profissional (2) }\end{array}$ & & & 0,34 & & 0,60 & & & 0,11 & & 0,05 \\
\hline Não & 29,7 & 1,00 & & 1,00 & & 28,8 & 1,00 & & 1,00 & \\
\hline $\operatorname{Sim}$ & 27,1 & $0,91(0,76-1,10)$ & & $0,94(0,76-1,17)$ & & 25,6 & $0,89(0,77-1,03)$ & & $0,85(0,72-1,00)$ & \\
\hline $\begin{array}{l}\text { Atividade profissional } \\
\text { na UBS (2) }\end{array}$ & & & $<0,001$ & & 0,76 & & & $<0,001$ & & 0,05 \\
\hline Médico & 40,1 & 1,00 & & 1,00 & & 47,7 & 1,00 & & 1,00 & \\
\hline Enfermeiro & 36,4 & $0,91(0,69-1,20)$ & & $0,99(0,71-1,39)$ & & 35,6 & $0,75(0,57-0,97)$ & & $0,85(0,62-1,16)$ & \\
\hline Outros de nível superior & 31,0 & $0,77(0,56-1,06)$ & & $0,94(0,65-1,34)$ & & 37,0 & $0,77(0,61-0,98)$ & & $0,88(0,65-1,19)$ & \\
\hline $\begin{array}{l}\text { Agentes Comunitários } \\
\text { de Saúde }\end{array}$ & 16,8 & $0,42(0,31-0,56)$ & & $0,49(0,28-0,85)$ & & 14,2 & $0,30(0,24-0,37)$ & & $0,53(0,34-0,82)$ & \\
\hline Outros de nível médio & 27,4 & $0,68(0,55-0,85)$ & & $0,74(0,47-1,15)$ & & 29,7 & $0,62(0,52-0,75)$ & & $1,01(0,69-1,48)$ & \\
\hline Primeiro emprego (2) & & & 0,35 & & 0,49 & & & 0,09 & & 0,73 \\
\hline Não & 27,6 & 1,00 & & 1,00 & & 27,9 & 1,00 & & 1,00 & \\
\hline Sim & 30,8 & $1,12(0,89-1,40)$ & & $1,10(0,84-1,44)$ & & 24,4 & $0,87(0,75-1,02)$ & & $1,04(0,85-1,27)$ & \\
\hline
\end{tabular}

(continua) 


\begin{tabular}{|c|c|c|c|c|c|c|c|c|c|c|}
\hline \multirow[t]{3}{*}{ Variáveis (nível) } & \multicolumn{5}{|c|}{ Região Sul } & \multicolumn{5}{|c|}{ Região Nordeste } \\
\hline & \multicolumn{3}{|c|}{ Análise bruta } & \multicolumn{2}{|c|}{ Análise ajustada } & \multirow[b]{2}{*}{$\%$} & \multicolumn{2}{|c|}{ Análise bruta } & \multicolumn{2}{|c|}{ Análise ajustada } \\
\hline & $\%$ & RP (IC95\%) & $\mathrm{p}$ & RP (IC95\%) & $p$ & & RP (IC95\%) & $p$ & RP (IC95\%) & $p$ \\
\hline Outro emprego além & & & $<0,001$ & & 0,001 & & & $<0,001$ & & 0,02 \\
\hline \multicolumn{11}{|l|}{ da UBS (2) } \\
\hline Não & 25,0 & 1,00 & & 1,00 & & 23,4 & 1,00 & & 1,00 & \\
\hline Sim & 38,7 & $1,55(1,29-1,86)$ & & $1,47(1,17-1,84)$ & & 40,2 & $1,73(1,50-1,99)$ & & $1,26(1,03-1,54)$ & \\
\hline Auto-avaliação da & & & $<0,001$ * & & 0,002 * & & & 0,004 * & & 0,001 * \\
\hline \multicolumn{11}{|l|}{ qualidade de vida (2) } \\
\hline Muito ruim & 66,7 & 1,00 & & 1,00 & & 16,7 & 1,00 & & 1,00 & \\
\hline Ruim & 48,2 & $0,72(0,36-1,44)$ & & $0,59(0,27-1,30)$ & & 50,0 & $3,0(0,49-1,83)$ & & $1,77(0,36-8,71)$ & \\
\hline Nem ruim nem boa & 32,0 & $0,48(0,26-0,88)$ & & $0,49(0,24-0,99)$ & & 27,3 & $1,64(0,27-9,85)$ & & $1,26(0,26-6,10)$ & \\
\hline Boa & 28,1 & $0,42(0,24-0,75)$ & & $0,45(0,23-0,90)$ & & 26,8 & $1,60(0,27-9,85)$ & & $1,16(0,24-5,59)$ & \\
\hline Muito boa & 21,7 & $0,32(0,17-0,61)$ & & $0,31(0,15-0,67)$ & & 21,3 & $1,28(0,21-7,78)$ & & $0,82(0,17-4,08)$ & \\
\hline
\end{tabular}

RP (IC95\%): razão de prevalência (intervalo de 95\% de confiança).

* Teste de Wald para tendência.

res que atuam com carga horária até 20 horas de trabalho na UBS, o que sugere fortemente atuação profissional em outro emprego, hipótese que se fortalece pela associação encontrada para a existência de outro emprego além da UBS e trabalho em regime precário no PSF.

Quando avaliado o uso de protocolos por parte do profissional de saúde, constata-se que aqueles que os usam são menos sedentários que os demais. No Brasil, os protocolos utilizados nas UBS, como para hipertensão e diabetes, incluem a prescrição da atividade física 19 . Nestes casos, o estudo de Ribeira et al. ${ }^{8}$, na Espanha, mostra que a promoção regular da atividade física é dependente do interesse pessoal e do conhecimento que o profissional tem dos benefícios da atividade física. Logo, a utilização dos protocolos colocaria estes profissionais em um grupo diferenciado por ressaltar os benefícios da atividade física para si e para os seus pacientes. Como uma das barreiras para a prática de atividade física pode ser o baixo conhecimento dos benefícios, a utilização de protocolos pode estar suprindo parte deste conhecimento necessário à modificação de comportamento 25 .

Algumas limitações do estudo devem ser consideradas, destacando-se as dificuldades para o estabelecimento de nexos causais em estudos transversais. É necessário ter cuidado na extrapolação dos resultados para outros grupos de trabalhadores, pois os trabalhadores em saúde têm algumas características específicas, como escolaridade mais elevada em comparação com outras profissões. Embora o estudo tenha apresentado perdas relativas ao preenchimento dos instrumentos pelos profissionais de saúde, os respondentes foram similares aos não respondentes no que diz respeito a sexo, idade e atividade profissional, situação que minimiza a possibilidade de viés de seleção. Algumas vantagens deste estudo também devem ser destacadas, entre elas a abrangência da investigação para um tema muito pouco explorado na literatura nacional e a lacuna existente sobre o tema até o momento. Outro ponto a ser destacado é a utilização de um questionário padronizado (IPAQ), que permite comparação com outros estudos nacionais e internacionais. Por fim, o tamanho de amostra garante um alto poder estatístico, mesmo para as análises estratificadas por modelo de atenção e região.

Podemos salientar que a atividade física ganha cada vez mais espaço como uma importante medida eficaz e de baixo custo para proporcionar melhores condições de vida às pessoas. Atualmente, existe um reconhecimento científico das possibilidades de ajuda terapêutica a diversas morbidades, e nos planos do Ministério da Saúde está a inclusão de profissionais de educação física no cotidiano da rede básica. A criação dos Núcleos de Apoio à Saúde da Família (NASF), pela Portaria $n^{\circ} .154$ do Ministério da Saúde 26, recomenda que a prática da atividade física seja o mais abrangente e coletiva possível, a fim de incluir os diversos grupos populacionais, adoecidos ou em maior risco, para que estes possam não só se reabilitar dos agravos já estabelecidos, como também prevenir problemas futuros. Contudo, ainda se verifica uma baixa prevalência de comportamento saudável em relação à atividade física entre os profissionais de saúde, o que pode resultar em pouca orientação aos pacientes e, 
Prevalência de sedentarismo, análise bruta e ajustada para profissionais de unidades básicas de saúde (UBS) conforme o modelo de atenção à saúde, 2005

\begin{tabular}{|c|c|c|c|c|c|c|c|c|c|c|}
\hline \multirow[t]{3}{*}{ Variáveis (nível) } & \multicolumn{5}{|c|}{ Programa Saúde da Família } & \multicolumn{5}{|c|}{ Tradicional } \\
\hline & \multicolumn{3}{|c|}{ Análise bruta } & \multicolumn{2}{|c|}{ Análise ajustada } & \multicolumn{3}{|c|}{ Análise bruta } & \multicolumn{2}{|c|}{ Análise ajustada } \\
\hline & $\%$ & RP (IC95\%) & $\mathrm{p}$ & RP (IC95\%) & $p$ & $\%$ & RP (IC95\%) & $\mathrm{p}$ & RP (IC95\%) & $p$ \\
\hline Sexo (1) & & & 0,03 & & 0,31 & & & 0,18 & & 0,73 \\
\hline Masculino & 30,0 & 1,00 & & 1,00 & & 33,8 & 1,00 & & 1,00 & \\
\hline Feminino & 24,8 & $0,83(0,70-0,98)$ & & $0,91(0,76-1,09)$ & & 29,6 & $0,87(0,72-1,06)$ & & $0,96(0,78-1,20)$ & \\
\hline Idade (anos) (1) & & & 0,44 * & & 0,83 * & & & 0,21 * & & 0,56 * \\
\hline $16-29$ & 26,0 & 1,00 & & 1,00 & & 26,7 & 1,00 & & 1,00 & \\
\hline $30-49$ & 24,5 & $0,94(0,80-1,11)$ & & $0,87(0,73-1,03)$ & & 31,2 & $1,16(0,92-1,47)$ & & $1,07(0,83-1,37)$ & \\
\hline 50 ou + & 33,2 & $1,28(1,02-1,59)$ & & $1,17(0,94-1,48)$ & & 31,6 & $1,17(0,88-1,57)$ & & $1,09(0,80-1,49)$ & \\
\hline Escolaridade (1) & & & $<0,001$ * & & 0,81 * & & & $<0,001$ * & & 0,002 * \\
\hline Fundamental incompleto & 27,8 & 1,00 & & 1,00 & & 21,6 & 1,00 & & 1,00 & \\
\hline Fundamental completo & 19,6 & $0,71(0,44-1,15)$ & & $0,87(0,51-1,49)$ & & 25,5 & $1,18(0,58-2,42)$ & & $1,30(0,60-2,83)$ & \\
\hline Médio incompleto & 18,7 & $0,67(0,44-1,03)$ & & $0,66(0,41-1,07)$ & & 22,0 & $1,02(0,50-2,08)$ & & $1,22(0,57-2,63)$ & \\
\hline Médio completo & 19,9 & $0,71(0,51-0,99)$ & & $0,74(0,51-1,07)$ & & 24,0 & $1,11(0,64-1,93)$ & & $1,17(0,63-2,16)$ & \\
\hline Superior incompleto & 26,6 & $0,96(0,64-1,43)$ & & $0,93(0,60-1,46)$ & & 25,3 & $1,17(0,62-2,21)$ & & $1,32(0,66-2,64)$ & \\
\hline Superior completo & 35,0 & $1,26(0,89-1,77)$ & & $0,93(0,59-1,44)$ & & 38,4 & $1,78(1,02-3,08)$ & & $1,76(0,94-3,32)$ & \\
\hline Pós-graduação & 37,4 & $1,35(0,97-1,87)$ & & $0,89(0,56-1,40)$ & & 41,7 & $1,93(1,12-3,33)$ & & $1,96(1,04-3,71)$ & \\
\hline Tabagismo (1) & & & 0,39 & & 0,10 & & & 0,76 & & 0,64 \\
\hline Não & 25,9 & 1,00 & & 1,00 & & 30,9 & 1,00 & & 1,00 & \\
\hline Sim & 28,5 & $1,10(0,89-1,36)$ & & $1,21(0,97-1,51)$ & & 29,5 & $0,96(0,71-1,28)$ & & $1,08(0,79-1,46)$ & \\
\hline Horas de trabalho cumpridas (2) & & & 0,07 * & & 0,63 * & & & $<0,001$ * & & 0,14 * \\
\hline Até 20 & 33,1 & 1,00 & & 1,00 & & 40,1 & 1,00 & & 1,00 & \\
\hline $21-40$ & 25,1 & $0,76(0,59-0,97)$ & & $0,89(0,66-1,21)$ & & 27,6 & $0,69(0,58-0,82)$ & & $0,82(0,64-1,03)$ & \\
\hline 40 ou mais & 30,4 & $0,92(0,56-1,51)$ & & $1,15(0,60-2,19)$ & & 30,4 & $0,76(0,40-1,43)$ & & $1,04(0,58-1,90)$ & \\
\hline $\begin{array}{l}\text { Tempo de trabalho na UBS } \\
\text { (meses) (2) }\end{array}$ & & & 0,37 * & & $0,21 *$ & & & 0,80 * & & 0,90 * \\
\hline Até 24 & 26,8 & 1,00 & & 1,00 & & 28,5 & 1,00 & & 1,00 & \\
\hline $25-108$ & 24,7 & $0,92(0,79-1,08)$ & & $0,90(0,76-1,08)$ & & 35,1 & $1,23(1,02-1,49)$ & & $1,24(1,01-1,53)$ & \\
\hline 109 ou + & 25,2 & $0,94(0,70-1,26)$ & & $0,86(0,60-1,23)$ & & 25,0 & $0,88(0,67-1,15)$ & & $0,88(0,64-1,20)$ & \\
\hline $\begin{array}{l}\text { Regime de trabalho } \\
\text { precarizado (2) }\end{array}$ & & & 0,003 & & 0,05 & & & 0,05 & & 0,94 \\
\hline Não & 27,8 & 1,00 & & 1,00 & & 32,7 & 1,00 & & 1,00 & \\
\hline Sim & 22,1 & $0,80(0,68-0,93)$ & & $0,85(0,72-1,00)$ & & 26,8 & $0,82(0,67-1,00)$ & & $0,99(0,79-1,25)$ & \\
\hline $\begin{array}{l}\text { Utilização de protocolos } \\
\text { pelo profissional (2) }\end{array}$ & & & 0,11 & & 0,07 & & & 0,43 & & 0,40 \\
\hline Não & 27,2 & 1,00 & & 1,00 & & 32,4 & 1,00 & & 1,00 & \\
\hline Sim & 24,1 & $0,87(0,77-1,03)$ & & $0,86(0,73-1,01)$ & & 30,2 & $0,93(0,78-1,11)$ & & $0,92(1,09-1,76)$ & \\
\hline Atividade profissional na UBS (2) & & & $<0,001$ & & 0,11 & & & $<0,001$ & & 0,62 \\
\hline Médico & 41,5 & 1,00 & & 1,00 & & 46,0 & 1,00 & & 1,00 & \\
\hline Enfermeiro & 34,3 & $0,83(0,65-1,06)$ & & $0,97(0,73-1,29)$ & & 40,5 & $0,88(0,64-1,21)$ & & $0,86(0,58-1,28)$ & \\
\hline Outros de nível superior & 35,3 & $0,85(0,65-1,11)$ & & $0,96(0,70-1,32)$ & & 34,0 & $0,74(0,56-0,97)$ & & $0,77(0,56-1,06)$ & \\
\hline $\begin{array}{l}\text { Agentes comunitários } \\
\text { de saúde }\end{array}$ & 14,4 & $0,35(0,28-0,43)$ & & $0,53(0,35-0,81)$ & & 16,9 & $0,37(0,27-0,51)$ & & $0,45(0,26-0,77)$ & \\
\hline Outros de nível médio & 29,1 & $0,70(0,58-0,85)$ & & $0,97(0,68-1,38)$ & & 28,5 & $0,62(0,50-0,76)$ & & $0,76(0,50-1,16)$ & \\
\hline Primeiro emprego (2) & & & 0,52 & & 0,05 & & & 0,32 & & 0,91 \\
\hline Não & 26,0 & 1,00 & & 1,00 & & 31,1 & 1,00 & & 1,00 & \\
\hline Sim & 24,6 & $0,95(0,80-1,12)$ & & $1,22(1,00-1,48)$ & & 28,1 & $0,90(0,74-1,11)$ & & $0,97(0,78-1,24)$ & \\
\hline $\begin{array}{l}\text { Outro emprego além } \\
\text { da UBS (2) }\end{array}$ & & & $<0,001$ & & 0,006 & & & $<0,001$ & & 0,02 \\
\hline Não & 23,2 & 1,00 & & 1,00 & & 25,5 & 1,00 & & 1,00 & \\
\hline Sim & 37,9 & $1,63(1,40-1,91)$ & & $1,32(1,08-1,60)$ & & 41,5 & $1,62(1,36-1,93)$ & & $1,33(1,04-1,68)$ & \\
\hline
\end{tabular}

(continua) 


\begin{tabular}{|c|c|c|c|c|c|c|c|c|c|c|}
\hline \multirow[t]{3}{*}{ Variáveis (nível) } & \multicolumn{5}{|c|}{ Programa Saúde da Família } & \multicolumn{5}{|c|}{ Tradicional } \\
\hline & \multicolumn{3}{|c|}{ Análise bruta } & \multicolumn{2}{|c|}{ Análise ajustada } & \multicolumn{3}{|c|}{ Análise bruta } & \multicolumn{2}{|c|}{ Análise ajustada } \\
\hline & $\%$ & RP (IC95\%) & $\mathrm{p}$ & RP (IC95\%) & $p$ & $\%$ & RP (IC95\%) & $\mathrm{p}$ & RP (IC95\%) & $p$ \\
\hline Auto-avaliação da qualidade & & & $<0,001$ * & & $<0,001 \times$ & & & 0,11 * & & 0,07 * \\
\hline \multicolumn{11}{|l|}{ de vida (2) } \\
\hline Muito ruim & 25,0 & 1,00 & & 1,00 & & 75,0 & 1,00 & & 1,00 & \\
\hline Ruim & 44,4 & $1,78(0,52-6,12)$ & & $1,30(0,38-4,42)$ & & 58,6 & $0,77(0,41-1,47)$ & & $0,80(0,40-1,63)$ & \\
\hline Nem ruim nem boa & 29,6 & $1,18(0,35-3,96)$ & & $1,00(0,30-3,33)$ & & 26,6 & $0,35(0,19-0,65)$ & & $0,47(0,24-0,93)$ & \\
\hline Boa & 25,2 & $1,00(0,30-3,36)$ & & $0,85(0,25-2,83)$ & & 31,1 & $0,41(0,23-0,74)$ & & $0,52(0,27-1,01)$ & \\
\hline Muito boa & 17,4 & $0,70(0,20-2,38)$ & & $0,59(0,17-2,05)$ & & 28,0 & $0,37(0,20-0,69)$ & & $0,44(0,22-0,90)$ & \\
\hline
\end{tabular}

RP (IC95\%): razão de prevalência (intervalo de 95\% de confiança).

* Teste de Wald para tendência.

conseqüentemente, oportunidades perdidas nas unidades básicas.

Urge a necessidade de estratégias para estimular os profissionais de saúde a uma mudança de comportamento. Neste sentido, cabe destacar a importância de projetos de capacitação de profissionais de saúde para a orientação e a prática de atividade física, a articulação entre profissionais de UBS e educadores físicos, e estudos de intervenção sobre a temática. Tais resultados também estimulam a pensar na possibilidade real da presença de profissional de educação física no grupo de profissionais que atendem a população, pela sua possibilidade de contribuir para a mudança de comportamento entre seus colegas profissionais e pelos benefícios que podem estender-se à população em geral que utiliza as UBS.

\section{Resumo}

Foi realizado um estudo transversal, em 240 unidades básicas de saúde (UBS) dos modelos tradicional de assistência ou vinculadas ao Programa Saúde da Família (PSF), envolvendo 3.347 profissionais de saúde, que responderam ao Questionário Internacional de Atividade Física. A prevalência de sedentarismo $(<150$ minutos por semana) foi de 27,5\% (IC95\%: 25,9-29,0). O sedentarismo foi mais freqüente nos profissionais do modelo tradicional, sendo tal diferença explicada pela presença dos agentes comunitários de saúde, cuja ocupação é bastante ativa apenas no modelo PSF. Houve maior percentual de sedentarismo nos profissionais que trabalham em municípios de grande porte e possuem alto nível sócio-econômico. Destaca-se a importância de projetos de capacitação de profissionais de saúde para a orientação e a prática de atividade física e a articulação entre profissionais de UBS e educadores físicos.

Atividade Motora; Pessoal de Saúde; Programa Saúde da Família

\section{Colaboradores}

F. C. V. Siqueira participou da concepção, análise dos dados, interpretação e redação final; participou também da elaboração dos instrumentos e processamento de dados. M. V. Nahas contribuiu com a concepção e revisão do artigo. L. A. Facchini revisou o artigo e coordenou o estudo. R. X. Piccini revisou o artigo e participou de todas as etapas da pesquisa. E. Tomasi colaborou na revisão do artigo. E. Thumé revisou o artigo e participou das diversas fases da pesquisa. D. S. Silveira contribuiu com a análise e a interpretação dos dados e revisou o artigo. P. C. Hallal participou da revisão do artigo desde sua concepção até a redação final.

\section{Agradecimentos}

Ministério da Saúde do Brasil e Banco Mundial pelo apoio financeiro. 


\section{Referências}

1. Pate RR, Pratt M, Blair SN, Haskell WL, Macera CA, Bouchard C, et al. Physical activity and public health. A recommendation from the Centers for Disease Control and Prevention and the American College of Sports Medicine. JAMA 1995; 273:402-7.

2. NIH Consensus Development Panel on Physical Activity and Cardiovascular Health. Physical activity and cardiovascular health. JAMA 1996; 276: 241-6.

3. Lee IM, Paffenbarger Jr. RS, Hsieh CC. Physical activity and risk of prostatic cancer among college alumni. Am J Epidemiol 1992; 135:169-79.

4. Shepard RJ. Physical activity, fitness and health: the current consensus. Quest 1995; 47:228-303.

5. Monteiro CA. Novos e velhos males da saúde no Brasil. A evolução do país e de suas doenças. São Paulo: Editora Hucitec/Universidade de São Paulo; 1995.

6. Organização das Nações Unidas. Plano de ação internacional para o envelhecimento 2002. Brasília Organização das Nações Unidas; 2003. (Série Institucional em Direitos Humanos, V1).

7. Facchini LA, Piccini RX, Tomasi E, Thumé E, Silveira DS, Siqueira FV, et al. Desempenho do PSF no Sul e no Nordeste do Brasil: avaliação institucional e epidemiológica da Atenção Básica a Saúde. Ciênc Saúde Coletiva 2006; 11:669-81.

8. Ribeira AP, McKenna J, Riddoch C. Attitudes and practices of physicians and nurses regarding physical activity promotion in the Catalan primary health-care system. Eur J Public Health 2005; 15:569-75.

9. Jatkinson J, Goody RB, Walter CA. Walking at work: a pedometer study assessing the activity levels of doctors. Scott Med J 2005; 50:73-4.

10. Fuscaldo JM. Prescribing physical activity in primary care. WV Med J 2002; 98:250-3.

11. Chakravarthy MV, Joyner MJ, Booth FW. An obligation for primary care physicians to prescribe physical activity to sedentary patients to reduce the risk of chronic health conditions. Mayo Clin Proc 2002; 77:165-73.

12. Venegas-Ochoa U, Llerenas-Tejeda C, AguayoGodinez A, Navarro-Solares JC, Corbala-Solares J, Campos A, et al. Physical activity and inactivity among female health service workers. Ginecol Obstet Mex 2006; 74:471-5.

13. Facchini LA, Piccini RX, Tomasi E, Thumé E, Teixeira VA, Silveira DS, et al. Avaliação de efetividade da Atenção Básica à Saúde em municípios das regiões Sul e Nordeste do Brasil: contribuições metodológicas. Cad Saúde Pública 2008; 24 Suppl 1:S159-72.

14. Ministério da Saúde. Termo de referência para o estudo de linha de base nos municípios selecionados para o componente 1 do PROESF. Brasília: Ministério da Saúde; 2004.
15. Craig CL, Marshall AL, Sjöström M, Bauman AE, Ainsworth BE, Pratt M, et al. International physical activity questionnaire: 12-country reliability and validity. Med Sci Sports Exerc 2003; 35:1381-95.

16. Hallal PC, Victora CG, Wells JC, Lima RC. Physical inactivity: prevalence and associated variables in Brazilian adults. Med Sci Sports Exerc 2003; 35:1894-900.

17. National Center for Chronic Disease Prevention and Health Promotion, Centers for Disease Control and Prevention, U.S. Department of Health and Human Services. Physical activity and health: a report of the Surgeon General. Atlanta: U.S. Department of Health and Human Services; 1996.

18. Departamento de Gestão e da Regulação do Trabalho em Saúde, Secretaria de Gestão do Trabalho e da Educação na Saúde, Ministério da Saúde. Trabalho precário no Sul. Brasília: Ministério da Saúde; 2006.

19. Departamento de Informação e Informática do SUS, Secretaria Executiva, Ministério da Saúde. Política Nacional de Informação e Informática em Saúde - proposta versão 2.0. Brasília: Ministério da Saúde; 2004.

20. Barros AJ, Hirakata VN. Alternatives for logistic regression in cross-sectional studies: an empirical comparison of models that directly estimate the prevalence ratio. BMC Med Res Methodol 2003; 3:21.

21. Victora CG, Huttly SR, Fuches SC, Olinto MT. The role of conceptual frameworks in epidemiological analysis: a hierarquical approachI. Int J Epidemiol 1997; 26:224-7.

22. Siqueira FV, Facchini LA, Piccini RX, Tomasi E, Thumé E, Silveira DS, et al. Atividade física em adultos e idosos residentes em áreas de abrangência de unidades básicas de saúde de municípios das regiões Sul e Nordeste do Brasil. Cad Saúde Pública 2008; 24:39-54.

23. Coordenação de Prevenção e Vigilância, Instituto Nacional de Câncer. Inquérito domiciliar sobre comportamentos de risco e morbidade referida de doenças e agravos não transmissíveis. Brasil, 15 capitais e Distrito Federal 2002 - 2003. Rio de Janeiro: Instituto Nacional de Câncer; 2004.

24. Trost SG, Owen N, Bauman AE, Sallis JF, Brown W. Correlates of adults' participation in physical activity: review and update. Med Sci Sports Exerc 2002; 34:1996-2001.

25. Garry JP, Diamond JJ, Whitley TW. Physical activity curricula in medical schools. Acad Med 2002; 77:818-20.

26. Ministério da Saúde. Portaria no ${ }^{\circ}$ 154, de 24 de janeiro de 2008. Diário Oficial de União 2008; 25 jan.

Recebido em 03/Dez/2008

Versão final reapresentada em 13/Mai/2009

Aprovado em 18/Mai/2009 